\title{
Right Atrial Electrocardiogram in the Analysis of Arrhythmias Following Acute Myocardial Infarction
}

\author{
D. E. JEWITT, Y. KISHON, M. THOMAS, AND J. P. SHILLINGFORD \\ From the M.R.C. Cardiovascular Research Unit, and the Department of Medicine, \\ Royal Postgraduate Medical School, Hammersmith Hospital, London W.12
}

The routine use of continuous cardiac monitoring in intensive coronary care units has demonstrated a high incidence of cardiac arrhythmias following acute infarction (Julian, Valentine, and Miller, 1964; Meltzer and Kitchell, 1966; Fluck et al., 1967; Lown et al., 1967). A prerequisite in assessing the value of therapeutic measures used to terminate any arrhythmia is that the arrhythmia and its prodromata should be precisely analysed. Using conventional electrocardiographic monitoring, this is not always possible since atrial activity may be poorly defined. In this situation right atrial electrocardiograms are of value and the technique has important advantages over oesophageal leads.

Direct intracavitary recordings were first reported by Hellerstein, Pritchard, and Lewis (1949), and recently Vogel et al. (1964) and Dreifus et al. (1965) have stressed the potential value of this technique at the bedside in the differentiation of complex arrhythmias.

This paper describes our experience of the value of the right atrial electrocardiogram in an intensive coronary care unit.

\section{SubJects AND MethodS}

Patients admitted to the intensive coronary care unit at Hammersmith Hospital are routinely monitored using press-stud chest electrodes as described by Fluck and Burgess (1966). Under sterile conditions a fine, pliable, nylon-coated, stainless steel wire (external diameter $0.063 \mathrm{~cm}$. and length $100 \mathrm{~cm}$.) is introduced percutaneously at the bedside into an antecubital vein by the Seldinger technique. An $80 \mathrm{~cm}$. length of fine polyethylene tubing (PE 60) is then passed into the vein over the nylon-coated guide wire so that the exposed tip of the wire lies within the polyethylene tubing. The external end of the insulated wire is then attached to the $\mathrm{V}$ lead terminal of a fully earthed multichannel

Received June 16, 1967.

7 electrocardiograph by a crocodile clip. The intravascular potentials are recorded simultaneously with a standard limb lead or monitoring chest lead, as the polyethylene tubing and wire within it are advanced together. As the PE 60 catheter passes from the superior vena cava into the right atrium the large amplitude $P$ waves with a sharp intrinsicoid deflection are clearly delineated from the QRS complexes (Fig. 1). Initially high in the right atrium the $\mathbf{P}$ wave is inverted, it becomes biphasic in mid-atrium and finally upright as the tricuspid valve is approached. It should not be necessary to pass across the tricuspid valve, but should this occur the presence of large amplitude QRS complexes with small $\mathbf{P}$ waves identifies the position and allows withdrawal back into the atrium.

In the absence of a significant arrhythmia at the time of admission, the insulated wire is removed and the PE 60 catheter left in situ with its tip in the right atrium and the external end taped to the forearm. This catheter is kept patent by intermittent flushing with small quantities of heparin and may be used if necessary for taking blood samples, pressure measurements, and injection of drugs. In the event of an arrhythmia subsequently developing with obscure atrial activity in the monitoring leads, the insulated wire is reintroduced into the polyethylene catheter to a point $5 \mathrm{~cm}$. from its tip, and the right atrial electrocardiogram is again recorded intermittently until the arrhythmia terminates.

\section{Results}

Illustrative recordings have been chosen from those made in patients admitted to the intensive care coronary unit since September 1966.

Differentiation of Tachycardias Associated with Abnormal $Q R S$ Complexes. The proof that an arrhythmia is ventricular in origin is accepted as being particularly difficult. This problem arises as both atrial and atrioventricular nodal arrhythmias may be associated with an abnormal QRS configuration due either to established intraventricular block 


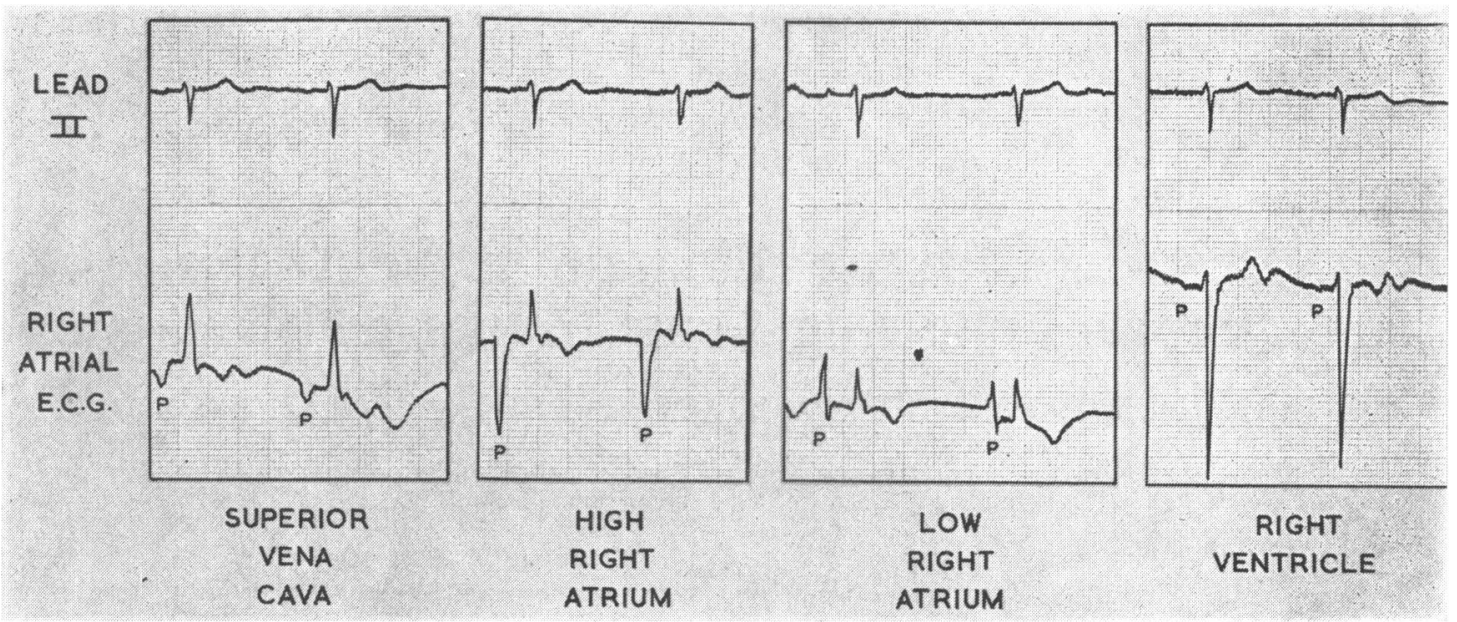

FIG. 1.-Normal intracavitary potentials. Simultaneous recordings of the intravascular potentials and standard lead II as the insulated wire within the PE 60 catheter is advanced from the superior vena cava into the atrium. The large amplitude $\mathbf{P}$ waves are initially inverted high in the atrium, becoming upright as the tricuspid valve is approached. In the right ventricle the QRS complexes are of large amplitude with smaller $P$ waves.

or aberrant conduction during the arrhythmia. The clear demonstration of atrial activity with the intra-atrial lead will allow a diagnosis of an ectopic atrial mechanism to be made. Thus atrial flutter may exist in the presence of established intraventricular block following myocardial infarction, and if flutter waves are not visible in a standard electrocardiogram it will simulate a ventricular or atrioventricular tachycardia. Fig. 2 illustrates how the atrial electrocardiogram established the diagnosis of atrial flutter in such a patient and allowed selection of appropriate therapy.

The differentiation of tachycardias of ventricular and low atrioventricular nodal origin, however, is more difficult, as has been stressed by Kistin (1966 a, b). The demonstration of clear atrial activity in the intra-atrial electrocardiogram may enable complete atrioventricular dissociation to be diagnosed in the presence of a tachycardia with abnormal QRS complexes, but this fails to differentiate between ventricular and low nodal arrhythmias with certainty. The presence of fusion or capture beats makes a ventricular arrhythmia more probable, though rare exceptions exist. In Fig. 3 a regular tachycardia with prolonged QRS complexes is seen in which the intra-atrial electrocardiogram clearly demonstrates complete atrioventricular dissociation. The presence of fusion beats, due to conduction of sinus impulses at about the same time as the ectopic ventricular focus discharges, is seen whenever a sinus $P$ wave falls 0.2 sec. before the $a b-$ normal QRS complex. This strongly favours a ventricular ectopic mechanism in this case.
Clear demonstration of atrial activity during an arrhythmia and after reversion to sinus rhythm may establish the presence of retrograde atrial activation from an atrioventricular or ventricular focus. Thus, in the standard electrocardiogram, the presence of aberration of the QRS complex in a patient with low atrioventricular nodal rhythm may mask the retrograde $P$ wave (Fig. 4). In this case a simultaneous intra-atrial recording showed retrograde $P$ waves deforming the late deflections of the QRS complex. The arrhythmia was, therefore, a low atrioventricular nodal rhythm with a QRS to retrograde $P$ wave interval of less than 0.1 sec., and not a ventricular tachycardia.

The onset and termination of an arrhythmia may also yield valuable information providing the $P$ waves and $Q R S$ complexes are clearly visible, as when an intra-atrial lead is available. Thus, if an arrhythmia begins with an ectopic premature $P$ wave it is supraventricular in origin, whether it is atrial or from high in the atrioventricular node. Conversely, if the arrhythmia begins with a QRS complex and retrograde $P$ waves are demonstrated, the presence of a $Q R S$ to retrograde $P$ interval of greater than $0.12 \mathrm{sec}$. favours a ventricular arrhythmia (Kistin, 1966a, b).

Analysis of Atrial Arrhythmias. The form of ectopic atrial activity seen in the intra-atrial electrocardiogram may be of value in differentiating between paroxysmal atrial tachycardia and atrial flutter (Fig. 5). In atrial flutter the intra-atrial record may be of two forms, as illustrated in Fig. 6; 


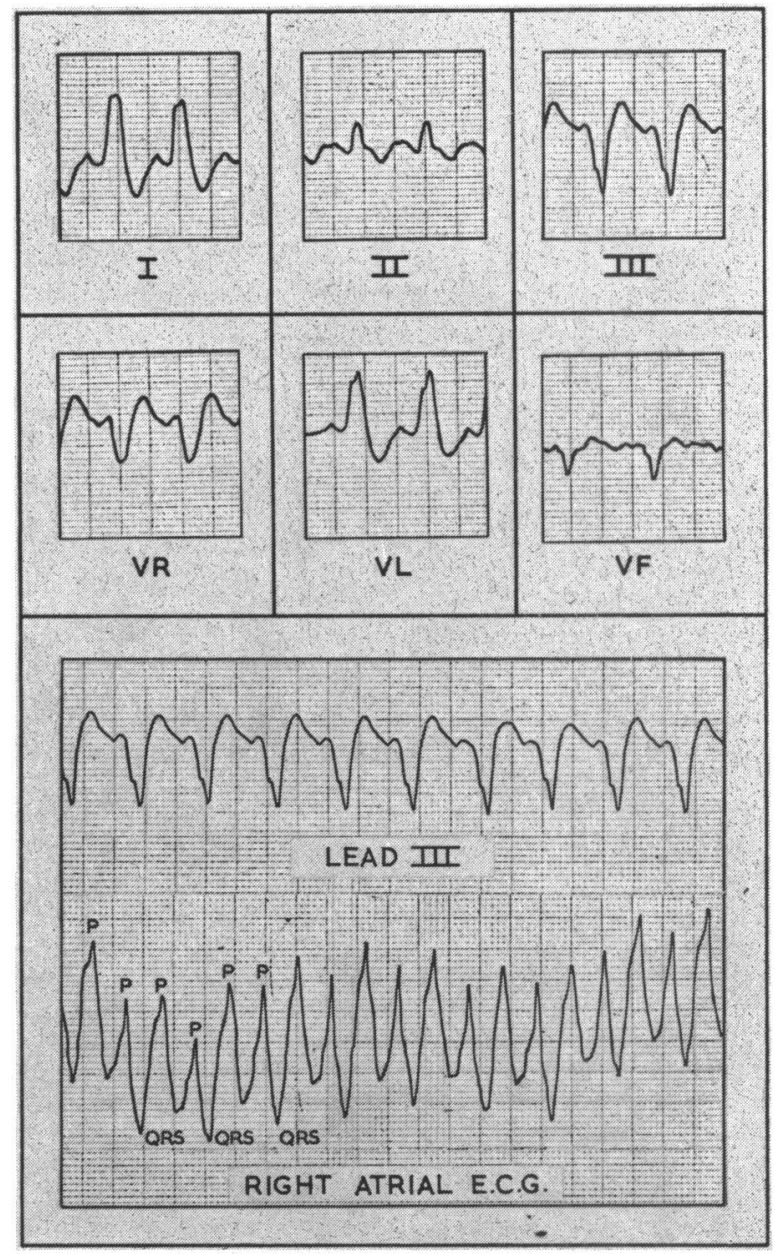

Fig. 2.-Atrial flutter. Above: standard limb leads in a patient with left bundle-branch block"and a regular tachycardia following acute infarction. Below: simultaneous recordings of lead III and the right atrial electrocardiogram. A regular tachycardia of 125 a minute without visible atrial activity in lead III but with regular flutter waves (p) at a rate of 250 a minute with $2: 1$ atrioventricular block in the atrial lead.

these are recordings made successively in the same intra-atrial position and were not associated with visible change in the simultaneous chest lead.

The appearance of atrial flutter fibrillation is seen in Fig. 7 in a patient who had an acute inferior lateral myocardial infarct. The atrial rhythm in this patient continuously changed from sinus rhythm to coupled atrial extrasystoles, atrial fibrillation, atrial flutter, and back to sinus rhythm. At the time of the recording in Fig. 7 complete atrioventricular dissociation was also present.

Diagnosis of Atrial Arrest. This rare arrhythmia is usually due to increased vagal tone associated with digitalis therapy. The diagnosis is only certain when an intra-atrial electrocardiogram excludes the presence of atrial activity. In Fig. 8 an example is shown from a patient with left bundle-branch block following an acute myocardial infarct, who was not receiving digitalis or $\beta$-adrenergic blocking drugs. The single sinus beat captures the slow idioventricular or nodal pacemaker and shows the appearance of the sinus $\mathbf{P}$ wave in this patient.

Atrioventricular Dissociation. Following myocardial infarction this arrhythmia may be due to a slowing of the sinus pacemaker, acceleration of a lower nodal or ventricular pacemaker, or an advanced degree of atrioventricular block. The demonstration of clear atrial activity which may only 


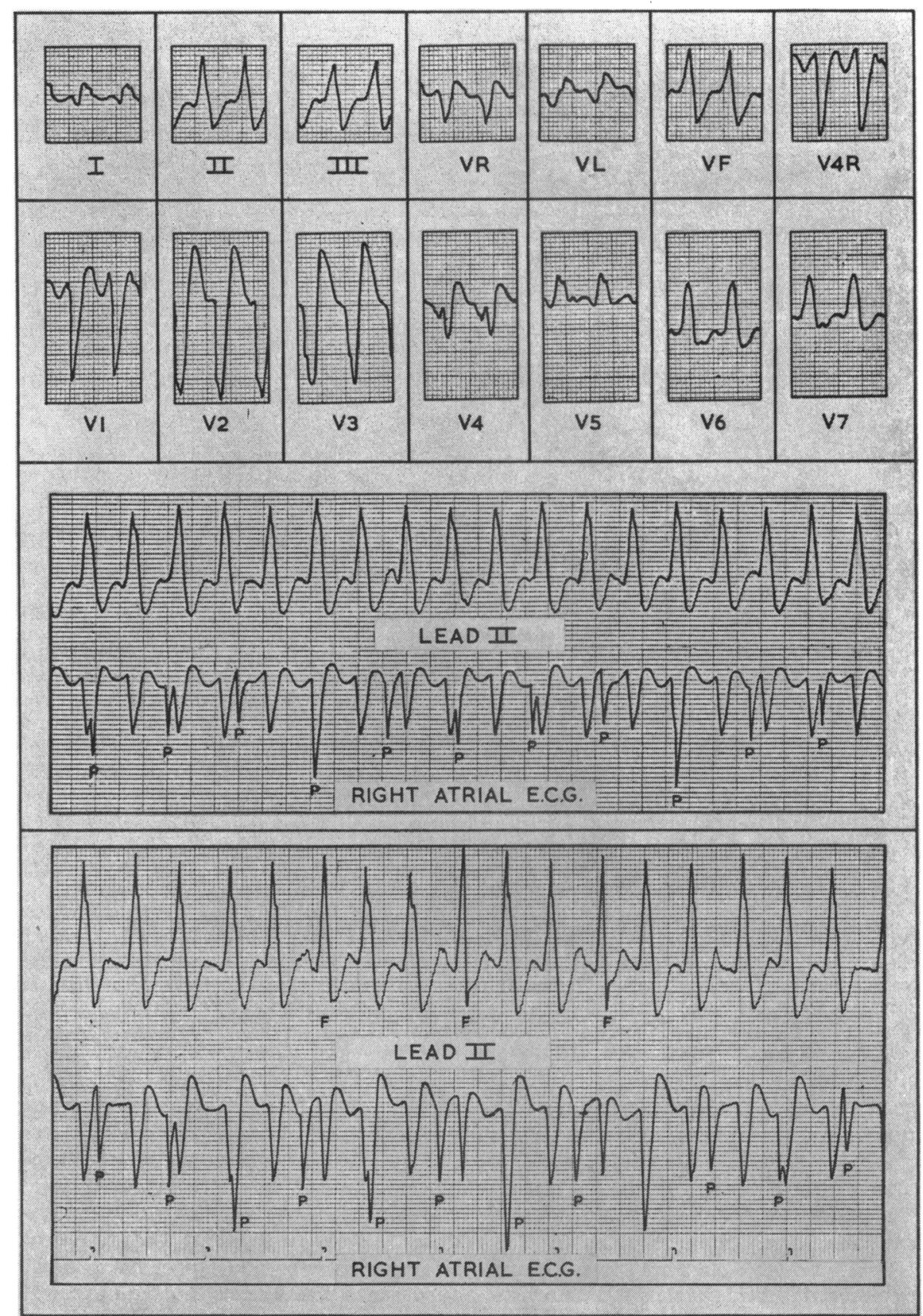

FIG. 3.-Ventricular tachycardia. Above: standard 12-lead electrocardiogram in a patient following a third myocardial infarct. Below: simultaneous recordings of lead II and the right atrial lead. A regular tachycardia at 166 a minute is seen in the 12-lead record without visible atrial activity and a left bundle-branch block pattern. The simultaneous atrial electrocardiograms show complete atrioventricular dissociation with a sinus rate of 100 a minute. In addition, fusion beats $(F)$ are seen in the lower record when a sinus $P$ wave falls $0 \cdot 2$ sec. before the ectopic ventricular complex. 


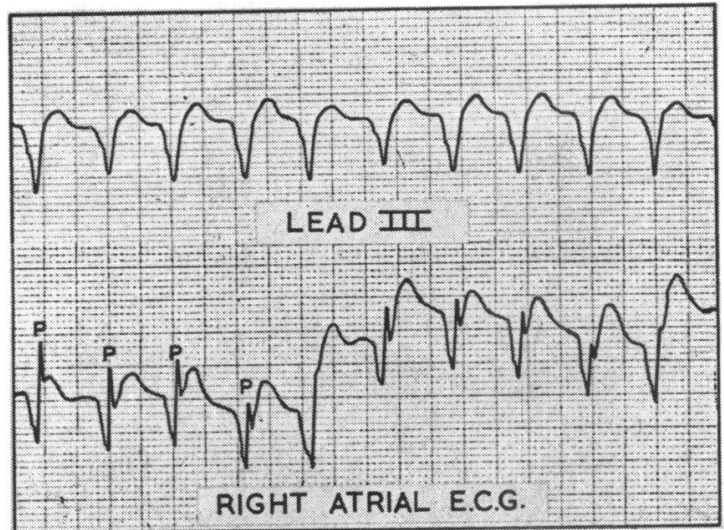

FIG. 4.-Low atrioventricular nodal tachycardia. Simultaneous recording of lead III and the right atrial electrocardiogram in a patient with left bundle-branch block following an acute infarct. A regular tachycardia at 136 a minute is seen in lead III and the simultaneous right atrial lead shows retrograde $P$ waves deforming the $Q R S$ complexes. The $Q R S$ to retrograde $P$ wave interval is 0.08 sec.

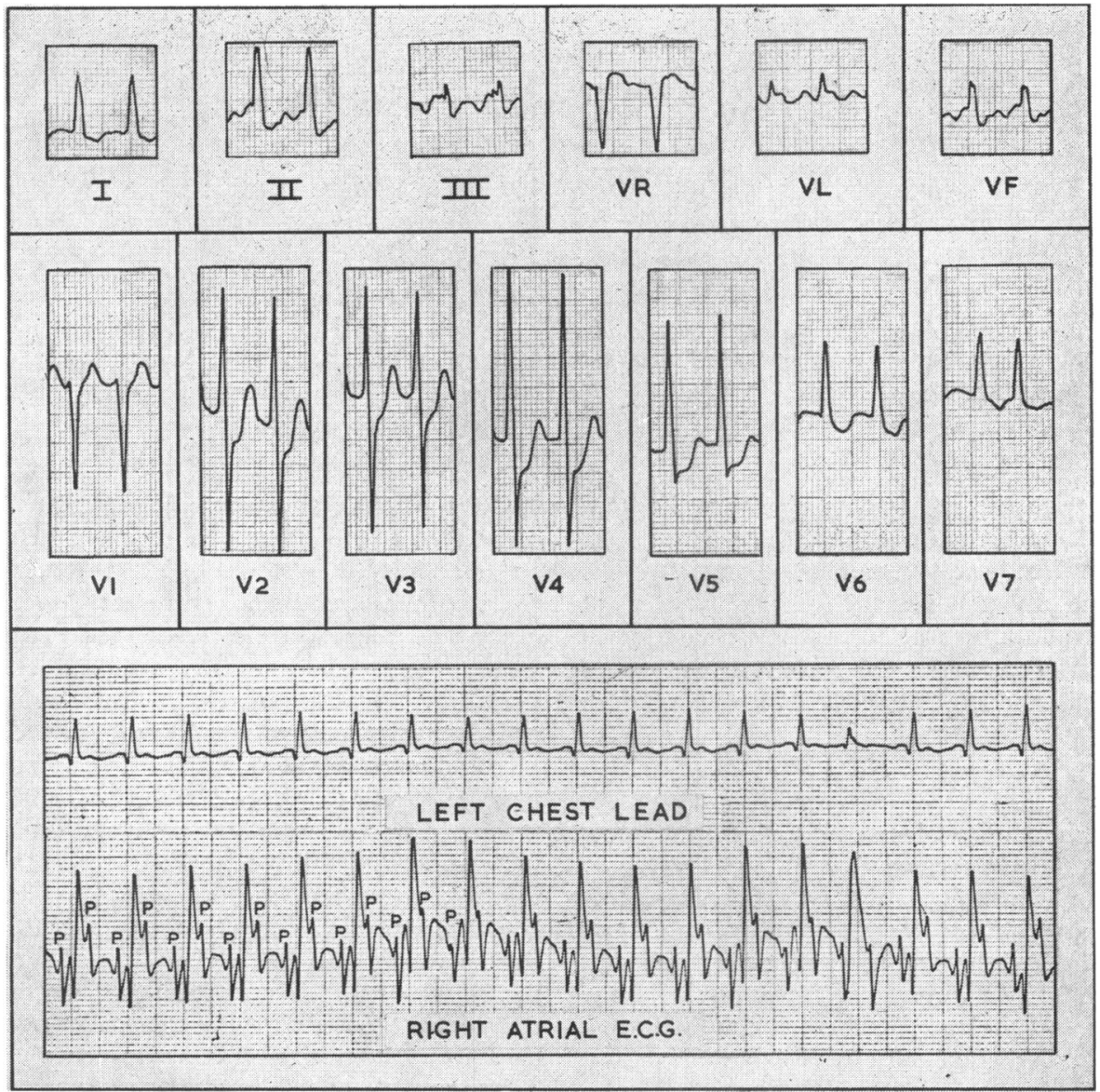

Fig. 5.-Atrial flutter. The 12-lead electrocardiogram above shows a regular tachycardia following acute subendocardial infarction in a known hypertensive with left ventricular hypertrophy. In the simultaneous strip of the left chest lead and intra-atrial lead, the latter shows flutter waves at a rate of 300 a minute with 2:1 atrioventricular block. 


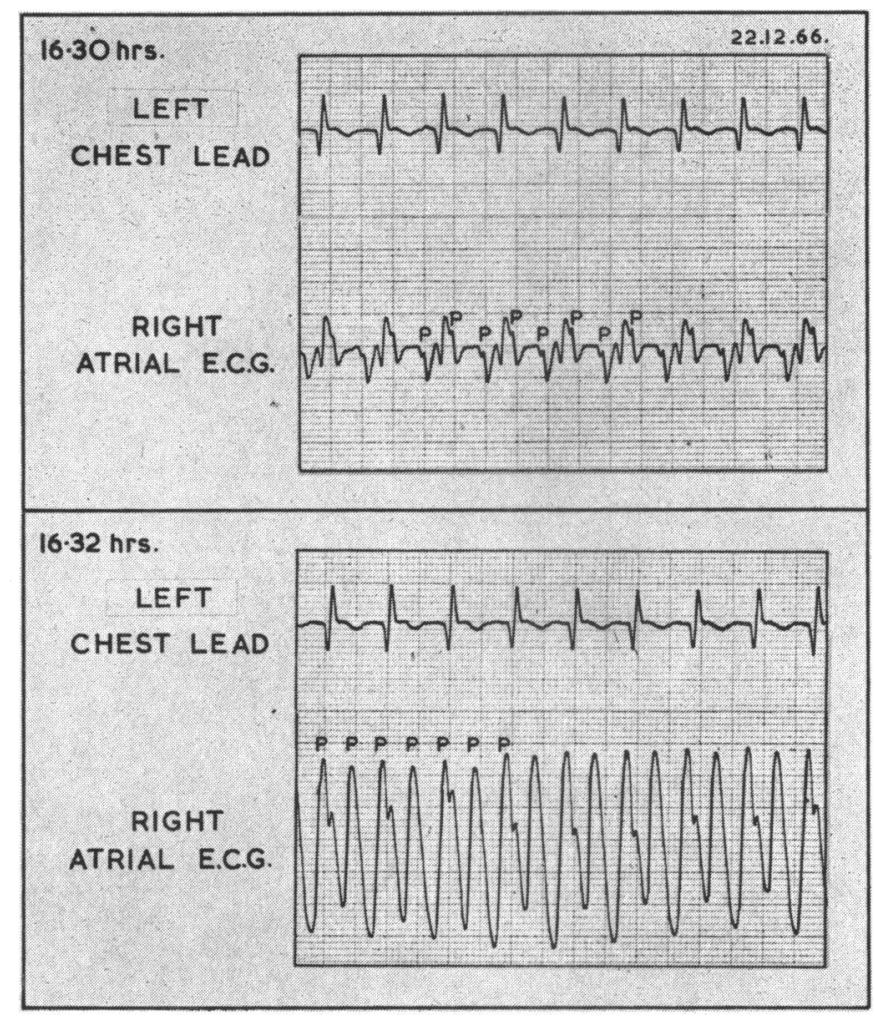

Fig. 6. - Two forms of flutter waves. Simultaneous left chest lead and right atrial lead. Atrial flutter is present in both, but at 16.30 hours the flutter waves are sharp and discrete. At 16.35 hours, in the same intra-atrial position, they are seen to be of large amplitude and of saw-tooth appearance deforming the QRS complexes.

be possible with the intra-atrial electrocardiogram allows this diagnosis to be made with certainty. In Fig. 3 complete atrioventricular dissociation is seen in a case of ventricular tachycardia, whereas in Fig. 7 the atrial flutter fibrillation is associated with complete atrioventricular dissociation, the nodal pacemaker discharging regularly at a rate of 65 a minute.

\section{Discussion}

The high incidence of cardiac arrhythmias now detected in intensive coronary care units has directed attention to their natural history and to available therapeutic procedures for their termination and suppression. Satisfactory assessment of different modes of therapy requires precise analysis of the arrhythmias and their prodromata. This is not an academic exercise, since only by clearly differentiating, for example, supraventricular from ventricular arrhythmias, can the appropriate therapy with drugs or DC cardioversion be selected and subsequent pharmacological suppression assessed.
Conventional electrocardiographic monitoring often fails to define the exact mechanism because atrial activity is obscured. In this situation the right atrial electrocardiogram is of value. As with oesophageal leads, a clearly defined $P$ wave is seen and the intra-atrial lead has the additional advantage that a high quality recording with a stable baseline can always be safely obtained without the attendant discomfort to the sick patient associated with the passage of an oesophageal tube.

The differentiation of tachycardias associated with abnormal QRS complexes remains the most valuable indication for its use. As illustrated in Fig. 2, atrial flutter may be confidently diagnosed in this situation. In 1966 Kistin pointed out that the demonstration of clear atrial activity often failed to differentiate between atrioventricular nodal tachycardia with abnormal QRS complexes and ventricular tachycardia. Thus the presence of complete atrioventricular dissociation, classically considered to indicate ventricular tachycardia, may also be present in a low atrioventricular nodal tachycardia. 


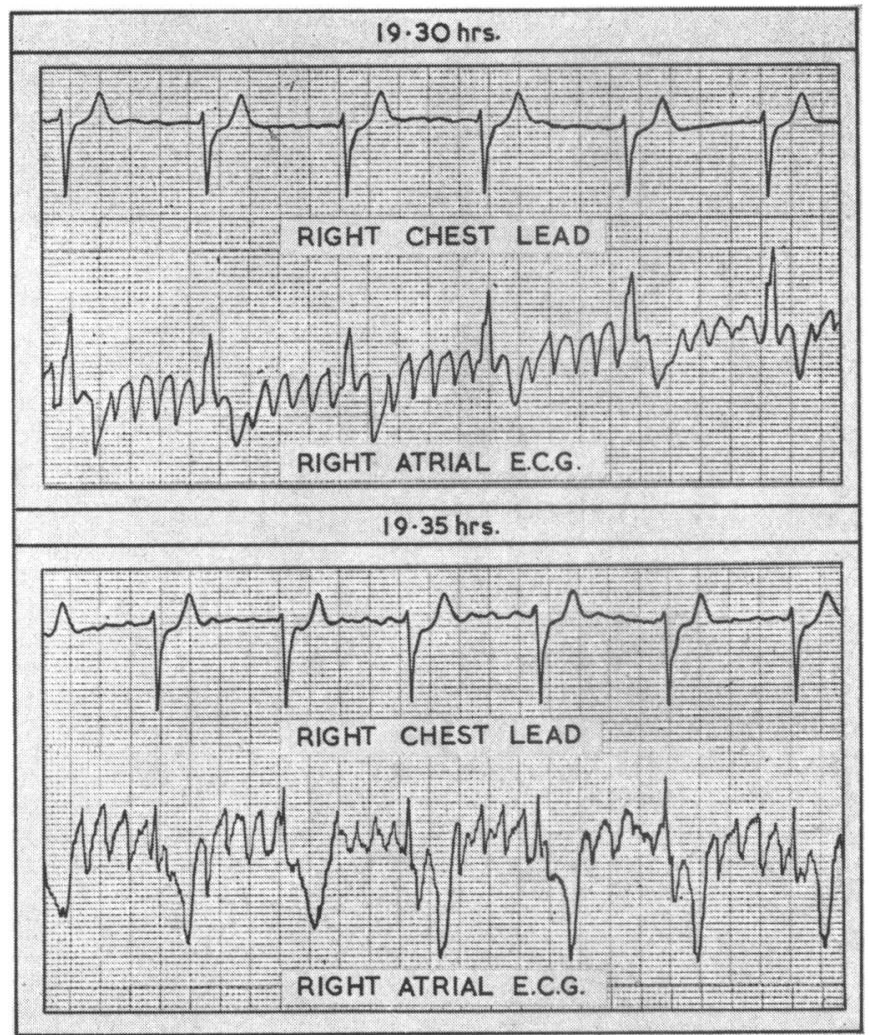

Fig. 7.-Atrial flutter fibrillation with atrio-ventricular dissociation. Simultaneous right chest lead and right atrial electrocardiograms. 19.30 hours: a regular rhythm at 65 a minute. The atrial rhythm is flutter fibrillation at a rate of 420 a minute with complete a-v dissociation and regular nodal rhythm. 19.35 hours : atrial fibrillation at 500 a minute with complete a-v dissociation and regular nodal rhythm at 67 a minute.

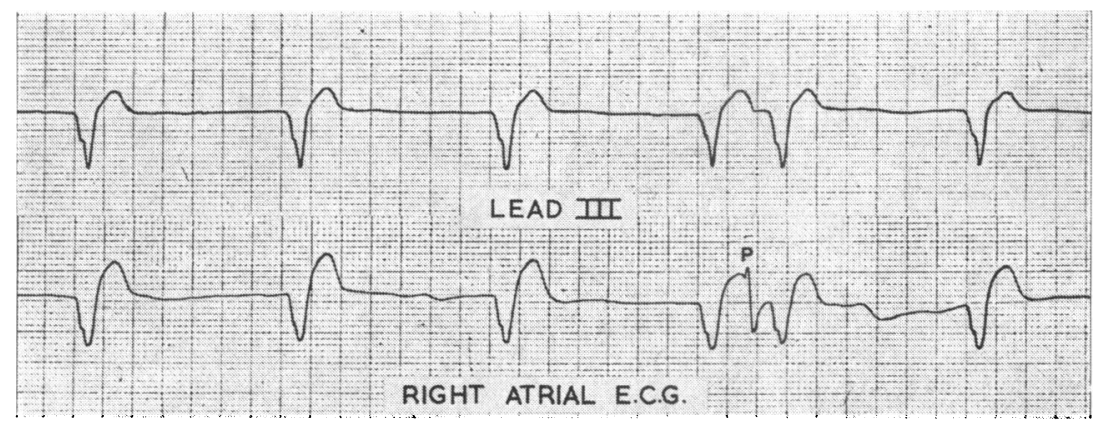

FIG. 8.-Atrial arrest with sinus capture. Simultaneous lead III and right atrial electrocardiogram in a patient with left bundle-branch block following acute infarction. Slow nodal rhythm at 43 a minute, with absent atrial activity until a sinus capture occurs and the $\mathbf{P}$ wave is clearly visible in the atrial electrocardiogram.

Similarly retrograde $P$ waves may occur in both arrhythmias, though as illustrated in Fig. 4 a short $Q R S$ to retrograde $P$ wave interval favours an atrioventricular nodal mechanism. The presence of fusion or capture beats may allow a presumptive diagnosis of ventricular tachycardia to be made, but rare exceptions to this statement have been recorded by Kistin (1966b). It is clear, therefore, that the diagnosis of ventricular tachycardia after acute myocardial infarction must be made only after precise and critical analysis of suggestive tracings has been made, and in arriving at this diagnosis multiple factors will need to be considered. The high incidence of ventricular tachycardia reported 
in many constant monitoring series may contain examples in which misdiagnosis occurred because of limited available information. Similarly the cases of ventricular tachycardia of short duration and favourable prognosis reported by Rothfeld et al. (1967), which were found to have slower ventricular rates than cases proceeding to ventricular fibrillation, may in some cases have been examples of nodal tachycardia with aberration of the QRS complexes.

The analysis of atrial arrhythmias, atrioventricular dissociation, all grades of sino-atrial and atrioventricular block, in addition to the site of origin of isolated ectopic beats, may be clarified by the intra-atrial lead. The subsequent natural history and response to appropriate therapy may then be accurately assessed.

Emergency endocardial pacing as a temporary measure is also feasible by advancing the tip of the insulated wire beyond the end of the PE 60 catheter and floating it without screening into the right ventricle. In this way lives may be saved that are lost in moving patients to screening units. Harris et al. (1965) first reported this technique.

In our hands right atrial electrocardiography has been safe in patients following acute myocardial infarction and no complications have occurred. By elucidating an obscure arrhythmia and allowing selection of appropriate therapy it may be a lifesaving technique.

\section{SUMMARY}

The use of a percutaneous, bedside right atrial electrocardiogram in an intensive coronary care unit is described.

The differentiation of tachycardias associated with abnormal ventricular complexes is illustrated.

The procedure is simple, safe, and without discomfort to the patient with an acute myocardial infarct.
We should like to thank the nursing staff for help and co-operation in this study. We are also grateful to Mr. Peter Burgess and Miss Jean Powell for technical assistance.

\section{REFERENCES}

Dreifus, L. S., Najmi, M., Pomerantz, D., and Novack, P. (1965). The right atrial electrogram: A bedside procedure for the diagnosis of cardiac arrhythmias. Dis. Chest, 48, 617.

Fluck, D. C., and Burgess, P. A. (1966). A press-stud electrode for continuous monitoring of the electrocardiogram. Lancet, 1, 1405.

-, Olsen, E., Pentecost, B. L., Thomas, M., Fillmore, S. J., Shillingford, J. P., and Mounsey, J. P. D. (1967). Natural history and clinical significance of arrhythmias after acute cardiac infarction. Brit. Heart f., 29, 170.

Harris, C. W., Hurlburt, J. C., Floyd, W. L., and Orgain, E. S. (1965). Percutaneous technic for cardiac pacing with a platinum-tipped electrode catheter. Amer. $\mathcal{F}$. Cardiol., 15, 48.

Hellerstein, H. K., Pritchard, W. H., and Lewis, R. L. (1949). Recording of intracavitary potentials through a single lumen, saline filled cardiac catheter. Proc. Soc. exp. Biol. (N.Y.), 71, 58.

Julian, D. G., Valentine, P. A., and Miller, G. G. (1964). Disturbances of rate, rhythm and conduction in acute myocardial infarction. Amer. F. Med., 37, 915.

Kistin, A. D. (1966a). Ventricular tachycardia and esophageal leads. In Mechanisms and Therapy of Cardiac Arrhythmias, ed. L. S. Dreifus and W. Likoff, p. 274. Grune and Stratton, New York.

- (1966b). Problems in the differentiation of ventricular arrhythmia from supraventricular arrhythmia with abnormal QRS. Progr. cardiovasc. Dis., 9, 1.

Lown, B., Fakhro, A. M., Hood, W. B., and Thorn, G. W. (1967). The coronary care unit. New perspectives and directions. F. Amer. med. Ass., 199, 188.

Meltzer, L. E., and Kitchell, J. B. (1966). The incidence of arrhythmias associated with acute myocardial infarction. Progr. cardiovasc. Dis., 9, 50.

Rothfeld, E. L., Bernstein, A., Parsonnet, V., Zucker, I. R., and Alinsonorin, C. A. (1967). Telemetric monitoring of the electrocardiogram in acute myocardial infarction. Dis. Chest, 51, 193.

Vogel, J. K. L., Tabari, K., Averill, K. H., and Blount, S. G. (1964). A simple technique for identifying $P$ waves in complex arrhythmias. Amer. Heart f., 67, 158. 